\title{
Success and Failure of Primary Medical, Nonoperative Management In Breast Cancer
}

\author{
Seraina Margaretha Schmid, MD¹, Aleksandra Anna Modlasiak, MD ${ }^{1}$, Mary Elizabeth Myrick, MD ${ }^{1}$, \\ Nerbil Kilic, MD ${ }^{1,2}$, Carsten Thomas Viehl, MD $^{3}$, Andreas Schötzau, MS $^{4}$, and Uwe Güth, MD ${ }^{1}$ \\ ${ }^{1}$ Department of Gynecology and Obstetrics, University Hospital Basel (UHB), Basel, Switzerland; ${ }^{2}$ Department of Medical \\ Oncology, University Hospital Basel (UHB), Basel, Switzerland; ${ }^{3}$ Department of Surgery, UHB, Basel, Switzerland; \\ ${ }^{4}$ Schötzau and Simmen Institute for Biomathematics, Basel, Switzerland
}

\begin{abstract}
Background. Nonoperative but systemic therapy as firstline management is offered to some patients with breast cancer (BC) who have assumed limited life expectancy, such as older women or those who have distant metastases at initial presentation. We evaluated rates of and predicting factors for success and failure of this therapy approach.

Methods. Seventy-five patients who were initially treated only systemically, and cases in which local control while avoiding surgery was the intended long-term therapy goal were analyzed. Additionally, two stage-dependent subgroups were distinguished (A: stage I-III, $n=31$; B: stage $\mathrm{IV}, n=44)$. Failure of therapy was defined as when secondary surgery had to be performed due to locoregional progression or in case of no surgery when severe locoregional clinical signs/symptoms were observed during the further course.
\end{abstract}

Results. Patients in group A were older than those in group B (81 vs. 67.5 years; $P<0.001)$ and showed an increased survival (5-year rates: $40.2 \%$ vs. $24.3 \%$ ). In 24 patients of the entire cohort (32\%), secondary surgery had to be performed; surgery was performed more often in group A $(58.1 \%$ vs. $13.6 \%)$. In the cases in which no surgery was performed $(n=51), 11$ women $(21.6 \%)$ suffered from severe locoregional symptoms in the palliative situation (A: $n=1 ; \mathrm{B}: n=10$ ). Although the presence of stage IV was a significant factor for therapy success (odds ratio (OR), 2.59; 95\% confidence interval (CI), 0.95-7.05;

(C) Society of Surgical Oncology 2011

First Received: 2 December 2010;

Published Online: 11 February 2011

U. Güth, MD

e-mail: ugueth@uhbs.ch
$P=0.039)$, skin involvement was associated with failure of therapy (OR, 3.57; 95\% CI, 1.16-11.11; $P=0.031)$.

Conclusions. Nonoperative treatment may be offered to selected patients with $\mathrm{BC}$ who have assumed limited life expectancy. These women must be openly informed that this approach is not successful in nearly half of the cases.

Surgery is the central component of curative treatment and for local control of breast cancer. There are, however, a great many situations in which surgery is not performed as initial treatment. Such situations occur when patients refuse surgery against medical advice or when the tumor is inoperable due to excessive locoregional spread or in the case when patients are deemed unfit for surgical intervention due to frailty or comorbidity. Furthermore, in some patients with distant metastatic disease at initial presentation, surgery is not considered to be beneficial when a limited survival time is expected. The impact of nonoperative management has been evaluated for several subgroups, such as older women, in case of patients' refusal, and women who present with stage IV breast cancer. ${ }^{1-14}$ These studies consistently showed that surgery is associated with improved local control and survival.

In our opinion, the differentiation made by other authors between the above-mentioned subgroups of patients is not necessarily sensible from a clinician's view and is partly artificial. ${ }^{1-6,8-14}$ If patients and physicians agree on nonoperative treatment as initial breast cancer management, the basic approach of this concept is to expect/hope for long-term control of locoregional disease with systemic therapy. In most cases, the decision for this treatment option is made with the assumption of a patient's short life expectancy. This is the case in older patients and/or patients with severe comorbidity, as well as in patients who 
are clearly in a palliative situation due to distant metastases. That this separation is artificial becomes apparent when one emphasizes the common goal of nonoperative management; we defined this therapy approach as successful where local control could be reached by palliative systemic therapy and no clinically relevant locoregional progression developed.

We recognized the similar conception of the nonoperative patient group and examined, on the basis of the data of a prospective Swiss breast cancer database during a 20year period, for the first time in literature, the group of patients in its entirety who were initially treated only systemically and without surgical intervention and highlight the conceptual similarities of these patients who are otherwise considerably heterogeneous in terms of age, clinical appearance, tumor characteristics, therapeutic options, prognosis, and individual perceptions. Thus, in addition to the entire group, it is still important to evaluate the above-mentioned stage-defined subgroups separately. Particularly, a comparison between these two subgroups has not been conducted before. Furthermore, we evaluate rates of and predicting factors for success and failure of this particular therapy approach.

\section{PATIENTS AND METHODS}

The prospective relational Basel Breast Cancer Database (BBCD) includes all newly diagnosed primary invasive breast cancer cases treated at the University Women's Hospital Basel, Switzerland, since 1990. Data were recorded continuously from the medical files. For this study, data from patients treated through 2009 were analyzed $(n=1,495)$.

It was the goal of our study to evaluate patients 1) who did not undergo surgery as initial therapy but were treated systemically and 2) where local control while avoiding surgery was the intended long-term therapy goal.

Therefore, patients who had neoadjuvant therapy ( $n=68 ; 4.6 \%$ of the entire BBCD cohort), i.e., where the therapy concept implied surgery after a defined period of preoperative systemic therapy, were excluded from analysis. Second, three women $(0.2 \%)$ who refused all therapy options, i.e., in which the natural course of breast cancer could be studied, were not considered. Third, we excluded 12 patients who initially refused an operation, started a systemic therapy but changed their opinion within 8 weeks, and underwent surgical intervention.

In the end, 75 nonoperative patients (5\% of the BBCD cohort) who met the aforementioned inclusion criteria comprise the cohort of this study. We provided complete outcome information for these women. They were followed until death or, if they remained alive and disease-free, for a maximum of 17 years. The median follow-up duration after breast cancer diagnosis was 25 (range, <1-206) months. At the time of data collection in November 2010, the outcome data of patients still alive was not older than 6 weeks.

For this study, information on tumor classification was reported according to the current AJCC/UICC TNM guidelines. $^{15,16}$ Twenty-eight patients showed skin involvement. From these, 21 had noninflammatory skin involvement (T4b) showing the classical clinical sign of ulceration, and 7 patients presented with inflammatory carcinoma. $^{17}$

In most cases $(n=69)$, the diagnosis of invasive carcinoma was confirmed by core needle biopsy; in six cases diagnosis was made by fine-needle aspiration biopsy. Hormonal receptor assay and assessment of histopathologic grading according to the Nottingham modification of the Scarff-Bloom-Richardson grading scheme also were performed (Table 1). Additional biological predictive factors (e.g., overexpression of HER-2/neu, p53, EGFR), which eventually may prove to be more reliable index measures of tumor aggressiveness, were not performed on a regular basis during the 1990s; therefore, information on these factors was not available for all patients and were not taken into account in the final analysis. Tumor size was assessed clinically (cT classification) by imaging and/or physical examination.

Each patient underwent a workup to determine staging, including a recording of clinical history, physical examination, routine blood studies and additional radiologic studies (e.g., chest x-ray, sonography of the liver, nuclear bone scans, computed tomography, magnetic resonance imaging) as needed to exclude metastatic disease. There was no standard nonoperative therapeutic approach during the study period (Table 2). Treatment was selected based on the individual situation taking comorbid conditions and patients' perceptions and compliance into consideration.

In this study, in addition to the entire study cohort $(n=75)$, we analyzed two subgroups, which were distinguished by the disease stage at initial presentation: (1) patients who did not show distant metastases (stage I-III; $n=31$ ); (2) patients who had distant metastases (stage IV; $n=44)$.

Furthermore, we analyzed how often the goal of nonoperative management was met and which factors were associated with a successful therapy. We defined the therapy goal as reached when the treatment managed to control the locoregional disease and long-term avoidance of surgery was assured. We defined the following situations as failure to meet the therapy goal:

- Secondary surgery had to be performed due to progressive disease 
TABLE 1 Clinicopathologic characteristics of 75 patients with breast cancer who had primary medical and nonoperative management as initial treatment
$E R$ estrogen receptor

\section{${ }^{a}$ AJCC/UICC TNM}

classification $^{15,16}$

b TX: occult carcinoma (TX N3 M1)

c In cases with inflammatory carcinoma, tumor size was not recorded

\begin{tabular}{|c|c|c|c|}
\hline Variable & $\begin{array}{l}\text { Entire study } \\
\text { cohort }\end{array}$ & $\begin{array}{l}\text { Group A } \\
\text { (stage I-III) }^{\text {a }}\end{array}$ & $\begin{array}{l}\text { Group B } \\
\text { (stage IV) }^{\mathrm{a}}\end{array}$ \\
\hline No. of patients & 75 & 31 & 44 \\
\hline \multicolumn{4}{|l|}{ Age at diagnosis $[y r]$} \\
\hline Median (range) & $73(38-92)$ & $81(52-92)$ & $67.5(38-92)$ \\
\hline \multicolumn{4}{|l|}{ TNM stage at initial diagnosis ${ }^{\mathrm{a}}$} \\
\hline I & $8(10.7)$ & $8(25.8)$ & - \\
\hline II & $15(20)$ & $15(48.4)$ & - \\
\hline III & $8(10.7)$ & $8(25.8)$ & - \\
\hline IV & $44(58.6)$ & - & 44 \\
\hline \multicolumn{4}{|c|}{ Tumor size at initial diagnosis, clinically measured [mm] } \\
\hline Mean/median (range) & $52.6 / 32(11-220)$ & $35.1 / 25(11-150)$ & $65.2 / 50(14-220)$ \\
\hline \multicolumn{4}{|l|}{ Tumor category, clinical classification ${ }^{\mathrm{a}}$} \\
\hline $\mathrm{TX}^{\mathrm{b}}$ & $1(1.3)$ & - & $1(2.2)$ \\
\hline T1c $(11-20 \mathrm{~mm})$ & $14(18.7)$ & $9(29)$ & $5(11.4)$ \\
\hline $\mathrm{T} 2(21-50 \mathrm{~mm})$ & $25(33.3)$ & $15(48.4)$ & $10(22.7)$ \\
\hline $\mathrm{T} 3(>50 \mathrm{~mm})$ & $7(9.3)$ & - & $7(15.9)$ \\
\hline $\mathrm{T} 4 \mathrm{~b}$ & $20(26.7)$ & $4(12.9)$ & $16(36.4)$ \\
\hline $\mathrm{T} 4 \mathrm{c}$ & $1(1.3)$ & - & $1(2.2)$ \\
\hline T4d (inflammatory carcinoma) ${ }^{\mathrm{c}}$ & $7(9.3)$ & $3(9.7)$ & $4(9.1)$ \\
\hline \multicolumn{4}{|l|}{ Histologic subtype } \\
\hline Ductal invasive & $56(74.7)$ & $24(74.4)$ & $32(72.7)$ \\
\hline Lobular invasive & $13(17.3)$ & $4(12.9)$ & $9(20.5)$ \\
\hline Other types & $6(8)$ & $3(9.7)$ & $3(6.8)$ \\
\hline \multicolumn{4}{|l|}{ Hormone receptor status } \\
\hline ER positive & $51(68.0)$ & $23(74.2)$ & $28(63.6)$ \\
\hline Not known & $6(8)$ & $3(9.7)$ & $3(6.8)$ \\
\hline \multicolumn{4}{|l|}{ Grading } \\
\hline G1/2: well/moderately differentiated & $36(48)$ & $15(48.4)$ & $21(47.7)$ \\
\hline G3: poorly differentiated & $26(34.7)$ & $10(32.3)$ & $16(36.4)$ \\
\hline Not known/not applicable & $13(17.3)$ & $6(19.3)$ & 7 (15.9) \\
\hline
\end{tabular}

- In case of no surgery, the development of advanced locoregional clinical morbidity that significantly impaired quality of life, such as large ulcerating and fungating lesions (to the point of cancer en cuirasse) or severe lymphedema.

Data collection methods and study design were approved by the institutional review board.

\section{Statistical Methods}

Using the Kaplan-Meier method, overall survival (OS) was calculated from the date of diagnosis to the date of death or the time of last follow-up for patients still living. Statistical differences between the survival curves of the study subgroups were analyzed using the log-rank test. To predict factors contributing to attain the therapy goal, logistic regression was performed. Comparisons between nominal parameters were made using the Fisher's exact test. Comparisons between metric parameters were made with the Wilcoxon rank-sum test. A $P$ value $<0.05$ was considered significant. Statistical analyses were performed with R Development Core Team software, version 2.11.1 (Vienna, Austria).

\section{RESULTS}

The clinicopathologic, treatment, and outcome characteristics of the 75 patients who had a nonoperative therapy approach are summarized in Tables 1 and 2. The median age of 73 years of these patients was 13 years older than that of the entire cohort of patients recorded in the BBCD; patients in group A were significantly older than those in group B (81 vs. 67.5 years; $P<0.001)$. Clinically assessed mean tumor size was $52.6 \mathrm{~mm}$ in the entire cohort; patients in group A had significantly smaller tumors (35.1 vs. $65.2 \mathrm{~mm} ; P=0.002)$. 
TABLE 2 Treatment and outcome characteristics of 75 patients with breast cancer who had primary medical and nonoperative management as initial treatment
$E C$ endocrine therapy; $C T$ chemotherapy, including trastuzumab; $N E D$ no evidence of disease

${ }^{a}$ Definition of last follow-up: (1) for patients who are alive: the status of the most previous consultation; (2) for patients who died: status in the prefinal situation

${ }^{b}$ For analysis of long-term survivors, only patients who were diagnosed before 2005, i.e., who had a potential observation time of at least 5 years, were included (entire cohort, $n=47$; group $\mathrm{A}$, $n=22$; group $\mathrm{B}, n=25$ )

\begin{tabular}{|c|c|c|c|}
\hline Variable & $\begin{array}{l}\text { Entire } \\
\text { study cohort }\end{array}$ & $\begin{array}{l}\text { Group A } \\
{\text { (stage I-III })^{1}}\end{array}$ & $\begin{array}{l}\text { Group B } \\
\text { (stage IV) }^{1}\end{array}$ \\
\hline Number of patients & 75 & 31 & 44 \\
\hline \multicolumn{4}{|l|}{ Primary medical therapy } \\
\hline Endocrine therapy (EC) & $48(64.0)$ & $28(90.3)$ & $20(45.5)$ \\
\hline Chemotherapy (CT) & $7(9.3)$ & - & $7(15.9)$ \\
\hline Combination: $\mathrm{EC}+\mathrm{CT}$ & $20(26.7)$ & $3(9.7)$ & $17(38.6)$ \\
\hline \multicolumn{4}{|l|}{ Duration of primary medical therapy } \\
\hline Mean/median [mo] (range) & $24.1 / 14(1-174)$ & $22.4 / 15(3-118)$ & $25.3 / 10(1-174)$ \\
\hline Initial positive response & $61(81.3)$ & $27(87.1)$ & $34(77.3)$ \\
\hline Secondary surgery performed & $24(32.0)$ & $18(58.1)$ & $6(13.6)$ \\
\hline $\begin{array}{l}\text { In the case no surgery was performed: } \\
\text { Locoregional situation at last follow-up }\end{array}$ & 51 & 13 & 38 \\
\hline No clinical signs & $27(52.9)$ & $11(84.6)$ & $16(42.1)$ \\
\hline Minor clinical signs & $13(25.5)$ & $1(7.7)$ & 12 (31.6) \\
\hline Major clinical signs & $11(21.6)$ & $1(7.7)$ & $10(26.3)$ \\
\hline \multicolumn{4}{|l|}{ Further clinical course } \\
\hline No morbidity & $27(36.0)$ & $18(58.1)$ & $9(20.5)$ \\
\hline Locoregional progression & $1(1.3)$ & $1(3.2)$ & - \\
\hline Systemic progression & $25(33.3)$ & $8(25.8)$ & 17 (38.6) \\
\hline Locoregional + systemic progression & $22(29.3)$ & $4(12.9)$ & $18(40.9)$ \\
\hline $\begin{array}{l}\text { Therapy goal reached: no surgery, no or only } \\
\text { minor locoregional clinical signs/symptoms } \\
\text { at last follow-up }\end{array}$ & $40(53.3)$ & $12(38.7)$ & $28(63.6)$ \\
\hline \multicolumn{4}{|l|}{ Outcome status } \\
\hline Died of metastatic disease & $43(57.3)$ & $11(35.5)$ & $32(72.7)$ \\
\hline Died of other causes & $15(20)$ & $13(41.9)$ & $2(4.5)$ \\
\hline Alive, NED & $6(8)$ & $5(16.1)$ & $1(2.2)$ \\
\hline Alive with breast cancer & $11(14.7)$ & $2(6.5)$ & $9(20.5)$ \\
\hline \multicolumn{4}{|l|}{ Survival time $(y r)$} \\
\hline$<1$ & $17(22.7)$ & $3(9.7)$ & $14(31.8)$ \\
\hline$>3^{b}$ & $25(53.2)$ & $16(72.7)$ & $9(36)$ \\
\hline$>5^{\mathrm{b}}$ & $19(40.4)$ & $11(50)$ & $8(25)$ \\
\hline$>10^{\mathrm{b}}$ & $5(10.6)$ & $4(18.2)$ & $1(4)$ \\
\hline
\end{tabular}

OS of the entire study cohort and the two subgroups is shown in Fig. 1; the 5- and 10-year adjusted survival rates of the entire cohort were $30.9 \%$ and $11.3 \%$, respectively. Compared with group B, the patients in group A had a significantly increased survival (5-year rates: $40.2 \%$ vs. $24.3 \%$; 10 -year rates: $16.1 \%$ vs. $7.3 \%$ ).

Duration of first-line systemic therapy was similar in both subgroups (22.4 vs. 25.3 months). Most patients responded positively after starting medical treatment (A: $87.1 \%$ vs. B: $77.3 \%$ ). In 24 patients of the entire cohort $(32 \%)$, a secondary surgery had to be performed due to locoregional progressive disease. Surgical intervention was performed more often in group A $(58.1 \%$ vs. $13.6 \%)$. In the cases where no surgery was performed, 11 women $(21.6 \%)$ suffered from severe locoregional symptoms and ailments in the palliative situation. Ten of these had stage IV disease at initial presentation (group B). Our defined therapy goal of nonoperative but systemic therapy as first-line management in breast cancer (no surgery; no or only minor locoregional clinical signs and symptoms at last follow-up) was met in approximately half of the patients. Despite this high local failure rate, approximately one-third of these patients achieved remission for more than 18 months by first-line therapy (data not shown).

The independent factors for a successful therapy also were analyzed by performing a multivariate analysis, which took into account tumor size, noninflammatory skin involvement, and metastatic disease at initial presentation (Table 3). Although the presence of stage IV (subgroup B) was a significant factor for therapy success (odds ratio 


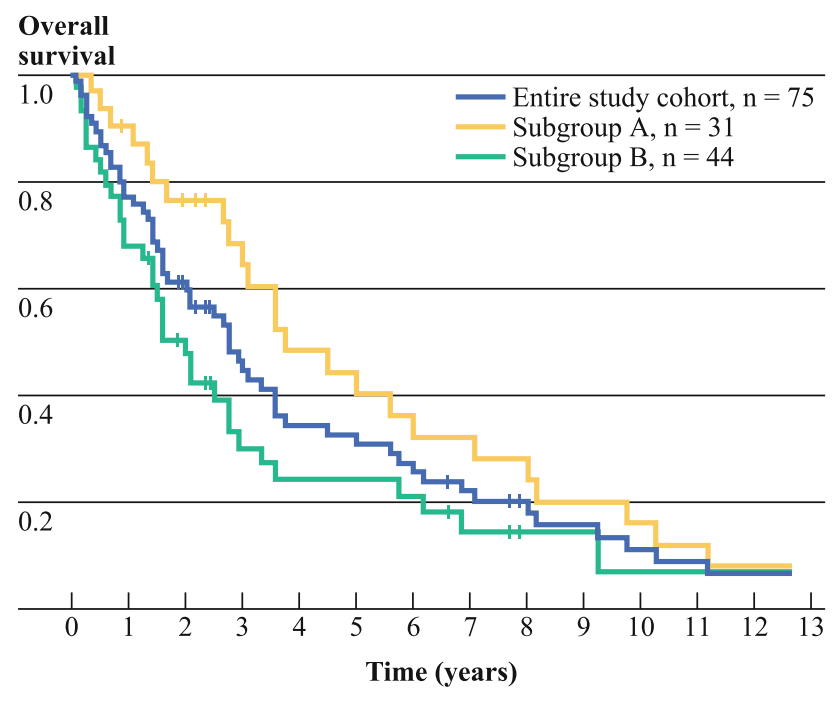

FIG. 1 Overall survival of 75 patients with breast cancer who had primary medical and nonoperative management as initial treatment. (1) entire study cohort, $n=75$; (2) subgroup A: disease stage I-III, $n=31$; (3) subgroup B: stage IV, $n=44$

TABLE 3 Multiple logistic regression predicting the factors that influence the successful attainment of the therapy goal in primary medical and nonoperative management as initial treatment (no surgery, no or only minor clinical signs/symptoms at last follow-up)

\begin{tabular}{lll}
\hline Variable & OR $(95 \%$ CI $)$ & $P$ value \\
\hline Tumor size & $0.58(0.31-1.08)$ & 0.162 \\
Non-inflammatory skin involvement & $0.28(0.09-0.86)$ & 0.031 \\
Stage IV at initial diagnosis & $2.59(0.95-7.05)$ & 0.004 \\
\hline
\end{tabular}

$O R$ odds ratio; $C I$ confidence interval

(OR), 2.59; 95\% confidence interval (CI), 0.95-7.05; $P=0.004)$, skin involvement was associated with failure of therapy (OR, 3.57; 95\% CI, 1.16-11.11; $P=0.031)$.

\section{DISCUSSION}

Atkin et al. reported reasons for a policy of nonoperative management in patients with nonmetastatic breast cancer and distinguished between the three categories: "inoperable" (1.3\% of their entire breast cancer cohort), "unfit for surgery" $(6 \%)$, and "refused surgery" $(1.5 \%){ }^{6}$ We think that this compartmentation is too rigid and that in most cases there is an overlap between the three categories. In our opinion, only very few carcinomas are actually inoperable. Even in most cases with locally advanced disease, surgical intervention is feasible. However, one must question whether rather complex reconstructive procedures to cover the chest wall defect are justified in every case. In our cohort, we considered only three cases to be inoperable. These were cases with a clinically assessed tumor size between 15 and $22 \mathrm{~cm}$; all showed noninflammatory skin involvement and one of them had additional deep infiltration of the chest wall (T4c). Furthermore, we think that by applying the current standards of anesthesia and postoperative surveillance today, it is possible for elderly and frail women to undergo safe breast surgery that has relatively little associated morbidity. ${ }^{18}$ When "refused surgery" is listed as a separated category for reasons in nonoperative management, one could assume that a patient's refusal for surgery is the result of a confrontation between patient and physician. This is not so in our experience. More often is the case that patients in oncological consultations tend to be wary and reluctant toward an operation. In further discussions, the physician can address the anxieties and reservations of the patients. Factors, such as average life expectancy, presence of comorbidities, and an analysis of potential treatment benefits versus risks of the proposed treatment strategy, must be carefully weighed and the option of a conservative nonoperative management can be offered to patients. ${ }^{19}$ It must be taken into account that, especially for older patients or those suffering from an incurable disease, "classical" hard medical facts, such as disease-specific and progression-free survival, are only one component of total care. Whereas physicians tend to focus on physical aspects, some patients and families view their situation with broader psychosocial and spiritual meaning, shaped by a lifetime of experiences. ${ }^{20}$ The priorities of these women often lie more with retaining their current life circumstances and their quality of life plays a more significant role in determining their treatment options rather than aiming solely for curative treatment. ${ }^{21}$ In our experience, in the majority of cases, nonoperative management is the result of shared decision-making and clear communication that acknowledges the values and preferences of patients and their families and not the patient's categorical refusal of medical advice. Before starting nonoperative management, it is usually agreed that the effect of systemic treatment should be monitored. An operation could still be considered in the case of subsequent locoregional tumor progression.

Randomized, clinical trials comparing surgery with tamoxifen in elderly nonmetastatic patients have shown that treatment solely with tamoxifen led to worse survival and failed to control the disease in most patients (45-81\%); thus, a high proportion required surgery after they developed local disease progression. ${ }^{1,2,4,5}$ In our study, we found a treatment failure of $61 \%$ in subgroup A. Most of these patients $(58 \%)$ required secondary surgery. Approximately one-third of these patients who had nonmetastatic disease at initial diagnosis died subsequently of breast cancer. This supports the assumption that residual or recurrent local disease could be a source of distant metastases. ${ }^{10}$ The conclusions from studies that evaluated nonoperative management in nonmetastatic breast cancer can be applied 
to patients with stage IV breast cancer; also in this situation, surgical treatment was found to be an independent factor associated with improved survival. ${ }^{8-14}$ To our knowledge, there is no study that has reported late surgery after initial nonoperative management in this group of patients. We found that the rate of $13.6 \%$ for late surgery in subgroup B was significantly lower than that of patients with nonmetastatic disease. However, one must strongly counter that further avoidance of surgical intervention in some patients in subgroup B led to the fact that $26.3 \%$ died not only from metastatic disease but also suffered from severe locoregional disease processes in the palliative situation. In accordance with Kenny et al., we found that uncontrolled locoregional relapse was a rare occurrence in subgroup A. ${ }^{4}$ In cases of uncontrolled locoregional progression, one must criticize the fact that the initial intention to perform surgery in subsequent tumor progression was not followed. One could assume that in metastatic patients the willingness of oncologists and/or surgeons to revise the nonoperative approach was low-to the disadvantage of the patients. We believe that even in an incurable situation, surgical intervention should be considered a useful palliation of agonizing pain and hygiene problems posed by bulky, necrotic tumors.

Life expectancy is an important factor in the decisionmaking process in the treatment of elderly and/or patients with metastatic breast cancer. ${ }^{19}$ This fact is mirrored in $42 \%$ of patients in subgroup A (which is comprised mostly of elderly women) who died of other causes than breast cancer. In approximately half of these patients, no secondary surgery was performed and medical treatment repressed or stabilized the disease, as intended, until death. However, an accurate estimation of life expectancy is difficult. In our study, only $9.7 \%$ of the patients in subgroup A showed a survival time $<12$ months (Table 2). This rate was considerably higher (31.8\%) in patients with distant metastases at initial presentation. Even when we restricted this analysis to women $\geq 75$ years who had died during the observation period (i.e., who had completed follow-up), the median follow-up time was 20.5 months in subgroup B and 36.5 months in group A. The shorter survival time might contribute to the fact that in patients of subgroup B a successful therapy goal was met more often.

At the other end of the spectrum of life expectancy are long-term survivors. Analyzing only patients who had been diagnosed up until 2005 , i.e., who had a possible observation time of at least 5 years (Table 2), $68 \%$ of the patients in subgroup A and $29 \%$ of patients in group B survived longer than 5 years. Five patients from our entire cohort even had a survival time longer than 10 years. One of these five patients was a woman who was diagnosed with stage IV breast cancer in 1995 who was treated with chemo- and endocrine therapy. The patient is still alive today (182 months after initial diagnosis) and has shown no evidence of disease for more than 10 years, neither in the nonoperated breast nor in the previously diagnosed metastatic sites (bone, lung). This is one of the very few cases, which refute the tenet that distant metastatic breast cancer is incurable in all cases. ${ }^{22}$

In our opinion, there is a clear role for surgery in preventing or palliating breast or chest wall symptoms, not only for older women and those with severe comorbid conditions, but also for patients in an incurable situation with distant metastases. Some authors prefer a surgical approach because it offers the maximum chance of local control and surgery may be more difficult after subsequent progression of disease. ${ }^{2,4}$ It is of great importance that women are entirely involved in the decision-making process concerning their treatment, and we acknowledge that they have the full right to choose not to undergo surgery. In accordance with other authors, we do not recommend "routine" breast surgery or definitive local treatment in all women but offer solely standard systemic palliative treatments for selected patients. ${ }^{1,5,9,10}$ These patients must openly be informed that a nonoperative approach is not successful in the nearly half of the cases and is generally associated with poorer outcome. In cases of overt locoregional progression, the preferred path, however, should be revised to avoid the complete loss of tumor control, which might be associated with severe symptoms and wound complications of breast and chest wall.

There are some limitations of our study that have to be addressed. First, we cover a long period of time in which therapy options have evolved considerably. Second, our study is retrospective. We do not believe, however, that this is necessarily a weak point. National Cancer Registries often exclude patients treated conservatively, limiting their validity for analysis in this group of patients. A clinical database of a single institution, such as the one used in this study, records data prospectively for all patients and provides solid follow-up data. The results in this series provide insights into patients who were treated with a nonoperative management. Future clinical trials are needed to further evaluate this subgroup of patients, which represents approximately $5-10 \%$ of all patients with breast cancer. In a field that is strongly shaped by very individual perceptions and decisions of partly opinionated patients, a randomized study is hardly possible.

\section{REFERENCES}

1. Fennessy M, Bates T, MacRae K, et al. Late follow-up of a randomized trial of surgery plus tamoxifen versus tamoxifen alone in women aged over 70 years with operable breast cancer. Br J Surg. 2004;91:699-704.

2. Fentiman IS, Christiaens MR, Paridaens R, et al. Treatment of operable breast cancer in the elderly: a randomised clinical trial 
EORTC 10851 comparing tamoxifen alone with modified radical mastectomy. Eur J Cancer. 2003;39:309-16.

3. Hind D, Wyld L, Beverley CB, Reed MW. Surgery versus primary endocrine therapy for operable primary breast cancer in elderly women (70 years plus). Cochrane Database Syst Rev 2006;CD004272.

4. Kenny F, Robertson J, Ellis I et al. Long-term follow-up of elderly patients randomized to primary tamoxifen or wedge mastectomy as initial therapy for operable breast cancer. Breast. 1998;7:335-9.

5. Mustacchi G, Ceccherini R, Milani S, et al. Tamoxifen alone versus adjuvant tamoxifen for operable breast cancer of the elderly: long-term results of the phase III randomized controlled multicenter GRETA trial. Ann Oncol. 2003;14:414-20.

6. Atkin GK, Scott MA, Wiggins JE, Callam MJ. The incidence, indications and outcome for the non-operative management of breast cancer. J Surg Oncol. 2007;96:137-43.

7. Verkooijen HM, Fioretta GM, Rapiti E, et al. Patients' refusal of surgery strongly impairs breast cancer survival. Ann Surg. 2005;242:276-80.

8. Babiera GV, Rao R, Feng L, et al. Effect of primary tumor extirpation in breast cancer patients who present with stage IV disease and an intact primary tumor. Ann Surg Oncol. 2006;13: 776-82.

9. Bafford AC, Burstein HJ, Barkley CR, et al. Breast surgery in stage IV breast cancer: impact of staging and patient selection on overall survival. Breast Cancer Res Treat. 2009;115:7-12.

10. Blanchard DK, Shetty PB, Hilsenbeck SG, Elledge RM. Association of surgery with improved survival in stage IV breast cancer patients. Ann Surg. 2008;247:732-8.

11. Fields RC, Jeffe DB, Trinkaus K, et al. Surgical resection of the primary tumor is associated with increased long-term survival in patients with stage IV breast cancer after controlling for site of metastasis. Ann Surg Oncol. 2007;14:3345-51.
12. Gnerlich J, Jeffe DB, Deshpande AD, et al. Surgical removal of the primary tumor increases overall survival in patients with metastatic breast cancer: analysis of the 1988-2003 SEER data. Ann Surg Oncol. 2007;14:2187-94.

13. Khan SA, Stewart AK, Morrow M. Does aggressive local therapy improve survival in metastatic breast cancer? Surgery. 2002;132:620-6; discussion 626-7.

14. Rapiti E, Verkooijen HM, Vlastos G, et al. Complete excision of primary breast tumor improves survival of patients with metastatic breast cancer at diagnosis. J Clin Oncol. 2006;24:2743-9.

15. Edge S, Byrd D, Compton C, et al. AJCC cancer staging manual. New York: Springer; 2009.

16. Sobin L, Gospodarowicz M, Wittekind C (2009) UICC TNM classification of malignant tumors Oxford. Wiley-Blackwell.

17. Guth U, Jane Huang D, Holzgreve W, et al. T4 breast cancer under closer inspection: a case for revision of the TNM classification. Breast. 2007;16:625-36.

18. Holmes C, Muss H. Breast cancer in older women. In: Singletary S, Robb G, Hortobagyi G (editors). Advanced therapy of breast cancer, 2nd ed. Hamilton: Decker 2004:727-42.

19. Rao VS, Garimella V, Hwang M, Drew PJ. Management of early breast cancer in the elderly. Int J Cancer. 2007;120:1155-60.

20. Steinhauser KE, Christakis NA, Clipp EC, et al. Factors considered important at the end of life by patients, family, physicians, and other care providers. JAMA. 2000;284:2476-82.

21. Yellen SB, Cella DF, Leslie WT. Age and clinical decision making in oncology patients. J Natl Cancer Inst. 1994;86:176670.

22. Guth U, Huang DJ, Dirnhofer S, et al. Distant metastatic breast cancer as an incurable disease: a tenet with a need for revision. Cancer J. 2009;15:81-6. 